\title{
Blow up of solutions for a class of fourth order nonlinear pseudo-parabolic equation with a nonlocal source
}

Huafei $\mathrm{Di}^{1,2}$ and Yadong Shang ${ }^{1,2^{*}}$

\section{"Correspondence:}

gzydshang@126.com

'School of Mathematics and

Information Science, Guangzhou

University, Guangzhou, 510006,

China

${ }^{2}$ Key Laboratory of Mathematics and Interdisciplinary Sciences of

Guangdong Higher Education,

Guangzhou, 510006, China

\begin{abstract}
In this paper, we consider the initial boundary value problem for a fourth order nonlinear pseudo-parabolic equation with a nonlocal source. By using the concavity method, we establish a blow-up result of the solutions under suitable assumptions on the initial energy.
\end{abstract}

MSC: 35B44; 35K30; 35K59

Keywords: blow up; fourth order; nonlinear pseudo-parabolic; nonlocal source; concavity method

\section{Introduction}

In this article, we are concerned with the following initial boundary value problem:

$$
\left\{\begin{array}{l}
u_{t}-\Delta u-\Delta u_{t}+\triangle^{2} u=u^{p}(x, t) \int_{\Omega} K(x, y) u^{p+1}(y, t) d y, \quad x \in \Omega, t>0, \\
u(x, 0)=u_{0}(x), \quad x \in \Omega, \\
u=\frac{\partial u}{\partial v}=0 \text { or } u=\Delta u=0, \quad x \in \partial \Omega, t>0,
\end{array}\right.
$$

where $p>0$, and $\Omega$ is a bounded domain of $\mathbb{R}^{n}(n \geq 1)$ with a smooth boundary $\partial \Omega$. Here, $v$ is the unit outward normal to $\partial \Omega$, and $K(x, y)$ is an integrable, real valued function such that $K(x, y)=K(y, x)$. It is well known that this type of equations describes a variety of important physical processes, such as the analysis of heat conduction in materials with memory, viscous flow in materials with memory [1], the theory of heat and mass exchange in stably stratified turbulent shear flow [2], the non-equilibrium water-oil displacement in porous strata [3], the aggregation of populations [4-6], the velocity evolution of ionacoustic waves in a collisionless plasma when ion viscosity is invoked [7], filtration theory $[8,9]$, cell growth theory $[10,11]$, and so on. In population dynamics theory, the nonlocal term indicates that evolution of species at a point of space does not depend only on the nearby density but also on the total amount of species due to the effects of spatial inhomogeneity; see [4].

There have also been many profound results on the existence of global solutions and asymptotic behavior of the solutions for the initial boundary value problems and the initial value problems of fourth order nonlinear pseudo-parabolic equations.

(c) $2015 \mathrm{Di}$ and Shang. This article is distributed under the terms of the Creative Commons Attribution 4.0 International License (http://creativecommons.org/licenses/by/4.0/), which permits unrestricted use, distribution, and reproduction in any medium, provided you give appropriate credit to the original author(s) and the source, provide a link to the Creative Commons license, and indicate if changes were made. 
In 1972, Kabanin [8] considered the following problem:

$$
\left\{\begin{array}{l}
u_{t}-\beta^{2} u_{x x t}+\gamma u_{x x x x}=\alpha u_{x x}, \quad 0<x<l, t>0, \\
u(x, 0)=\varphi(x), \quad 0 \leq x \leq l, \\
u(0, t)=u(l, t)=u_{x x}(0, t)=u_{x x}(l, t)=0, \quad 0 \leq t \leq T,
\end{array}\right.
$$

where $\alpha, \beta, \gamma$ are positive constants. A classical solution of this mixed problem is obtained through the Fourier method in the form of a series. Conditions sufficient for uniform convergence of this series are found.

In 1978, Bakiyevich and Shadrin [9] considered the following problem:

$$
\left\{\begin{array}{l}
u_{t}-\gamma u_{x x t}+\beta u_{x x x x}=\alpha u_{x x}+f(t, x), \quad x \in \mathbb{R}, t>0, \\
u(x, 0)=\varphi(x), \quad x \in \mathbb{R}
\end{array}\right.
$$

where $\alpha>0, \beta \geq 0, \gamma>0$ are constants. They showed that the solutions of this problem are expressed through the sum of convolutions of functions $\varphi(x)$ and $f(t, x)$ with corresponding fundamental solutions of the problem.

Zhao and Xuan [12] studied the following fourth order pseudo-parabolic equation:

$$
u_{t}-\alpha u_{x x}-\gamma u_{x x t}+\beta u_{x x x x}+f(u)_{x}=0, \quad x \in \mathbb{R}, t \geq 0 .
$$

They obtained the existence of the global smooth solutions for the initial value problem of (1.4) and discussed the convergence behavior of solutions as $\beta \rightarrow 0$.

Recently, Khudaverdiyev and Farhadova [13] discussed the following fourth order semilinear pseudo-parabolic equation:

$$
u_{t}-\alpha u_{x x t}+u_{x x x x}=f\left(t, x, u, u_{x}, u_{x x}, u_{x x x}\right), \quad 0 \leq x \leq 1,0 \leq t \leq T,
$$

with Ionkin type non-self-adjoint mixed boundary conditions, where $\alpha>0$ is a fixed number. They proved the local existence for a generalized solution of the mixed problem under consideration by combining generalized contracted mapping principle and Schauder's fixed point principle and then proved the global existence for a generalized solution by means of Schauder's stronger fixed point principle.

The so-called viscous Cahn-Hilliard equation is also in a class of fourth order nonlinear pseudo-parabolic equations and can be considered as a special case of (1.5). In recent years, a lot of attention has been paid to the viscous Cahn-Hilliard equations. For more and deeper investigations of the stability analysis (as $t \rightarrow \infty$ ) and the asymptotic behavior of viscous Cahn-Hilliard models, we refer readers to $[14,15]$ and the references therein.

Since the study on blow-up solutions for nonlinear parabolic equation with nonlocal source by Levine in [16], many efforts have been made devoted to the study of blow-up properties for nonlocal semilinear parabolic equations. The upper bound and lower bound of the blow-up time, blow-up rate estimate, blow-up set, and blow-up profile of the blowup solutions for a various of nonlocal semilinear parabolic equations with nonlocal source terms or nonlocal boundary condition have been widely studied in the last few decades; we refer the readers to [17-29] and the references cited therein.

Korpusov [30] considered a Sobolev type equation with a nonlocal source and obtained blow-up results under suitable conditions on initial data and nonlinear function. In [31], 
Bouziani studied the solvability of nonlinear pseudo-parabolic equation with a nonlocal boundary condition. More results on the global well-posedness for the nonlinear pseudoparabolic equation with nonlocal source can be found in [1] and the references therein.

Motivated by the above-mentioned works, we investigate the blow-up behavior of solutions of the initial boundary value problem for a fourth order nonlinear pseudo-parabolic equation with a nonlocal source (1.1). By using the concavity method, we prove a finite time blow-up result under some assumption on the initial energy $E(0)$.

\section{Preliminaries}

In this section, we first state a local existence theorem, which can be obtained by FaedoGalerkin methods. The interested readers are referred to Lions [32] or Escobedo and Herrero [33] for details.

Theorem 2.1 Assume that $p>0$ and $u_{0} \in H_{0}^{2}(\Omega)$. Then there exists a $T_{m}>0$ for which problem (1.1) has a unique local solution $u \in C^{1}\left(\left[0, T_{m}\right) ; H_{0}^{2}(\Omega)\right)$ satisfying

$$
\left(u_{t}, v\right)+(\nabla u, \nabla v)+\left(\nabla u_{t}, \nabla v\right)+(\Delta u, \Delta v)=\left(u^{p}(x, t) \int_{\Omega} K(x, y) u^{p+1}(y, t) d y, v\right)
$$

for all $v \in H_{0}^{2}(\Omega)$ and $t \in\left[0, T_{m}\right)$.

Before stating our principal theorem, we note that the Fréchet derivative $f_{u}$ of the nonlinear function $f(u)=u^{p}(x, t) \int_{\Omega} K(x, y) u^{p+1}(y, t) d y$ is

$$
\begin{aligned}
f_{u} \cdot h(x, t)= & p u^{p-1}(x, t) h(x, t) \int_{\Omega} K(x, y) u^{p+1}(y, t) d y \\
& +(p+1) u^{p}(x, t) \int_{\Omega} K(x, y) u^{p}(y, t) h(y, t) d y, \quad \forall u \in H^{2}(\Omega) .
\end{aligned}
$$

Clearly $f_{u}$ is symmetric and bounded, so that the potential $F$ exists and is given by

$$
\begin{aligned}
F(u) & =\int_{0}^{1}(f(\rho u), u) d \rho \\
& =\int_{0}^{1} \int_{\Omega} \rho^{p} u^{p}(x, t)\left[\int_{\Omega} K(x, y) \rho^{p+1} u^{p+1}(y, t) d y\right] u(x, t) d x d \rho \\
& =\frac{1}{2 p+2} \int_{\Omega} \int_{\Omega} K(x, y) u^{p+1}(x, t) u^{p+1}(y, t) d x d y .
\end{aligned}
$$

Now, differentiating the identity (2.2) with respect to $t$, it follows that

$$
\begin{aligned}
\frac{d}{d t} F(u)= & \frac{1}{2 p+2} \frac{d}{d t} \int_{\Omega} \int_{\Omega} K(x, y) u^{p+1}(x, t) u^{p+1}(y, t) d x d y \\
= & \frac{1}{2} \int_{\Omega} \int_{\Omega} K(x, y) u^{p}(x, t) u^{p+1}(y, t) u_{t}(x, t) d x d y \\
& +\frac{1}{2} \int_{\Omega} \int_{\Omega} K(x, y) u^{p}(y, t) u^{p+1}(x, t) u_{t}(y, t) d x d y \\
= & \int_{\Omega} \int_{\Omega} K(x, y) u^{p}(x, t) u^{p+1}(y, t) u_{t}(x, t) d x d y=\left(f(u), u_{t}\right),
\end{aligned}
$$

where we have used the symmetry of $K(x, y)$. 
To obtain the blow-up result, we will introduce the energy function. We have

$$
\begin{aligned}
E(t)= & \frac{1}{2} \int_{\Omega}|\nabla u|^{2} d x+\frac{1}{2} \int_{\Omega}|\Delta u|^{2} d x \\
& -\frac{1}{2 p+2} \int_{\Omega} \int_{\Omega} K(x, y) u^{p+1}(x, t) u^{p+1}(y, t) d x d y .
\end{aligned}
$$

Lemma 2.1 Let $p>0$ and $u$ be a solution of the problem (1.1). Then $E(t)$ is non-increasing function, that is, $E^{\prime}(t) \leq 0$. Moreover, the following energy equality holds:

$$
E(t)+\int_{0}^{t}\left(\left|u_{t}\right|^{2}+\left|\nabla u_{t}\right|^{2}\right) d x d \tau=E(0)
$$

Proof Multiplying (1.1) by $u_{t}$ and integrating over $\Omega$, we have

$$
\begin{aligned}
& \int_{\Omega}\left|u_{t}\right|^{2} d x+\int_{\Omega}\left|\nabla u_{t}\right|^{2} d x+\frac{1}{2} \frac{d}{d t} \int_{\Omega}|\nabla u|^{2} d x+\frac{1}{2} \frac{d}{d t} \int_{\Omega}|\Delta u|^{2} d x \\
& =\int_{\Omega} u^{p}(x, t)\left[\int_{\Omega} K(x, y) u^{p+1}(y, t) d y\right] u_{t}(x, t) d x .
\end{aligned}
$$

Hence, from (2.3), we obtain

$$
\begin{gathered}
\int_{\Omega}\left|u_{t}\right|^{2} d x+\int_{\Omega}\left|\nabla u_{t}\right|^{2} d x+\frac{1}{2} \frac{d}{d t} \int_{\Omega}|\nabla u|^{2} d x+\frac{1}{2} \frac{d}{d t} \int_{\Omega}|\Delta u|^{2} d x \\
=\frac{1}{2 p+2} \frac{d}{d t} \int_{\Omega} \int_{\Omega} K(x, y) u^{p+1}(x, t) u^{p+1}(y, t) d x d y
\end{gathered}
$$

and

$$
\int_{\Omega}\left|u_{t}\right|^{2} d x+\int_{\Omega}\left|\nabla u_{t}\right|^{2} d x+\frac{d}{d t} E(t)=0 .
$$

Integrating (2.5) from 0 to $t$, we find

$$
E(t)+\int_{0}^{t} \int_{\Omega}\left(\left|u_{t}\right|^{2}+\left|\nabla u_{t}\right|^{2}\right) d x d \tau=E(0)
$$

The proof of the Lemma 2.1 is completed.

\section{Blow up of solutions}

Now, we will state the blow-up result of the solutions to the problem (1.1).

Theorem 3.1 Assume that $p>0$ and $u_{0} \in H_{0}^{2}(\Omega)$. If $u(x, t)$ is a solution of the problem $(1,1)$ and the initial data $u_{0}(x)$ satisfies

$$
\int_{\Omega}\left(\left|u_{0}\right|^{2}+\left|\nabla u_{0}\right|^{2}\right) d x>\eta E(0)
$$

then the solution of problem (1.1) blows up in finite time; that is, the maximum existence time $T_{\max }$ of $u(x, t)$ is finite and

$$
\lim _{t \rightarrow T_{\max }^{-}} \int_{0}^{t} \int_{\Omega}\left(|u|^{2}+|\nabla u|^{2}\right) d x d \tau=+\infty
$$


where $\eta=\frac{\alpha}{m} ; m=\left(\frac{\alpha}{2}-1\right) \lambda_{1} ; 2 \leq \alpha \leq 2 p+2 ; \lambda_{1}$ is the first eigenvalue of operator $-\triangle$ under homogeneous Dirichlet boundary conditions.

Proof The proof makes use of the so-called 'concavity method'. Multiplying (1.1) by $u$ and integrating over $\Omega$, we have

$$
\begin{gathered}
\frac{1}{2} \frac{d}{d t} \int_{\Omega}|u|^{2} d x+\frac{1}{2} \frac{d}{d t} \int_{\Omega}|\nabla u|^{2} d x+\int_{\Omega}|\nabla u|^{2} d x+\int_{\Omega}|\Delta u|^{2} d x \\
\quad=\int_{\Omega} u^{p}(x, t)\left[\int_{\Omega} K(x, y) u^{p+1}(y, t) d y\right] u(x, t) d x .
\end{gathered}
$$

Hence

$$
\begin{aligned}
\frac{1}{2} \frac{d}{d t} & \int_{\Omega}|u|^{2} d x+\frac{1}{2} \frac{d}{d t} \int_{\Omega}|\nabla u|^{2} d x+\int_{\Omega}|\nabla u|^{2} d x+\int_{\Omega}|\Delta u|^{2} d x \\
& -\int_{\Omega} \int_{\Omega} K(x, y) u^{p+1}(x, t) u^{p+1}(y, t) d x d y+\alpha E(u)-\frac{\alpha}{2} \int_{\Omega}|\nabla u|^{2} d x \\
& -\frac{\alpha}{2} \int_{\Omega}|\Delta u|^{2} d x+\frac{\alpha}{2 p+2} \int_{\Omega} \int_{\Omega} K(x, y) u^{p+1}(x, t) u^{p+1}(y, t) d x d y \\
= & \frac{1}{2} \frac{d}{d t}\left[\int_{\Omega}|u|^{2} d x+\int_{\Omega}|\nabla u|^{2} d x\right]+\alpha E(u) \\
& +\left(\frac{\alpha}{2 p+2}-1\right) \int_{\Omega} \int_{\Omega} K(x, y) u^{p+1}(x, t) u^{p+1}(y, t) d x d y \\
& +\left(1-\frac{\alpha}{2}\right) \int_{\Omega}|\nabla u|^{2} d x+\left(1-\frac{\alpha}{2}\right) \int_{\Omega}|\Delta u|^{2} d x=0 .
\end{aligned}
$$

We consider the following function:

$$
H(t)=\int_{\Omega}\left(|u|^{2} d x+|\nabla u|^{2}\right) d x-\eta E(0)
$$

From (3.2), (3.3), Lemma 2.1, and Poincaré's inequality, we have

$$
\begin{aligned}
& \frac{1}{2} \frac{d}{d t} H(t) \\
&=\frac{1}{2} \frac{d}{d t} \int_{\Omega}\left(|u|^{2}+|\nabla u|^{2}\right) d x \\
&=\left(\frac{\alpha}{2}-1\right) \int_{\Omega}\left(|\nabla u|^{2}+|\Delta u|^{2}\right) d x-\alpha E(u) \\
& \quad+\left(1-\frac{\alpha}{2 p+2}\right) \int_{\Omega} \int_{\Omega} K(x, y) u^{p+1}(x, t) u^{p+1}(y, t) d x d y \\
&=\left(\frac{\alpha}{2}-1\right) \int_{\Omega}\left(|\nabla u|^{2}+|\Delta u|^{2}\right) d x-\alpha E\left(u_{0}\right)+\alpha \int_{0}^{t} \int_{\Omega}\left(\left|u_{t}\right|^{2}+\left|\nabla u_{t}\right|^{2}\right) d x d \tau \\
& \quad+\left(1-\frac{\alpha}{2 p+2}\right) \int_{\Omega} \int_{\Omega} K(x, y) u^{p+1}(x, t) u^{p+1}(y, t) d x d y \\
& \geq\left(\frac{\alpha}{2}-1\right) \int_{\Omega}\left(|\nabla u|^{2}+|\Delta u|^{2}\right) d x-\alpha E\left(u_{0}\right)
\end{aligned}
$$




$$
\begin{aligned}
& \geq\left(\frac{\alpha}{2}-1\right) \lambda_{1} \int_{\Omega}\left(|\nabla u|^{2}+|u|^{2}\right) d x-\alpha E\left(u_{0}\right) \\
& =m\left[\int_{\Omega}\left(|\nabla u|^{2}+|u|^{2}\right) d x-\eta E\left(u_{0}\right)\right]=m H(t),
\end{aligned}
$$

where $\eta=\frac{\alpha}{m} ; m=\left(\frac{\alpha}{2}-1\right) \lambda_{1} ; 2 \leq \alpha \leq 2 p+2 ; \lambda_{1}$ is the first eigenvalue of operator $-\Delta$ under homogeneous Dirichlet boundary conditions.

Due to the conditions (3.1), it follows that

$$
H(0)=\int_{\Omega}\left(\left|u_{0}\right|^{2}+\left|\nabla u_{0}\right|^{2}\right) d x-\eta E\left(u_{0}\right)>0 .
$$

Multiplying (3.4) by $e^{-2 m t}$, we have

$$
e^{-2 m t} \frac{d}{d t} H(t)-2 m e^{-2 m t} H(t)=\frac{d}{d t}\left[e^{-2 m t} H(t)\right] \geq 0 .
$$

From the last inequality above and (3.5), we obtain

$$
H(t) \geq H(0) e^{2 m t}>0
$$

From what has been discussed above, we find

$$
\frac{1}{2} \frac{d}{d t} \int_{\Omega}\left(|u|^{2}+|\nabla u|^{2}\right) d x>\alpha \int_{0}^{t} \int_{\Omega}\left(\left|u_{t}\right|^{2}+\left|\nabla u_{t}\right|^{2}\right) d x d \tau
$$

Now we define

$$
G(t)=\int_{0}^{t} \int_{\Omega}\left(|u|^{2}+|\nabla u|^{2}\right) d x d \tau
$$

Differentiating the identity (3.8) with respect to $t$, we deduce that

$$
\begin{aligned}
& G^{\prime}(t)=\int_{\Omega}\left(|u|^{2}+|\nabla u|^{2}\right) d x, \\
& G^{\prime \prime}(t)=\frac{d}{d t} \int_{\Omega}\left(|u|^{2}+|\nabla u|^{2}\right) d x \geq 2 \alpha \int_{0}^{t} \int_{\Omega}\left(\left|u_{t}\right|^{2}+\left|\nabla u_{t}\right|^{2}\right) d x d \tau,
\end{aligned}
$$

so we have

$$
\begin{aligned}
G^{\prime \prime}(t) G(t) \geq & 2 \alpha \int_{0}^{t} \int_{\Omega}\left(\left|u_{t}\right|^{2}+\left|\nabla u_{t}\right|^{2}\right) d x d \tau \cdot \int_{0}^{t} \int_{\Omega}\left(|u|^{2}+|\nabla u|^{2}\right) d x d \tau \\
\geq & 2 \alpha \int_{0}^{t} \int_{\Omega}\left|u_{t}\right|^{2} d x d \tau \cdot \int_{0}^{t} \int_{\Omega}|u|^{2} d x d \tau \\
& +2 \alpha \int_{0}^{t} \int_{\Omega}\left|u_{t}\right|^{2} d x d \tau \cdot \int_{0}^{t} \int_{\Omega}|\nabla u|^{2} d x d \tau \\
& +2 \alpha \int_{0}^{t} \int_{\Omega}\left|\nabla u_{t}\right|^{2} d x d \tau \cdot \int_{0}^{t} \int_{\Omega}|u|^{2} d x d \tau \\
& +2 \alpha \int_{0}^{t} \int_{\Omega}\left|\nabla u_{t}\right|^{2} d x d \tau \cdot \int_{0}^{t} \int_{\Omega}|\nabla u|^{2} d x d \tau .
\end{aligned}
$$


Using Schwarz's inequality, we get

$$
\begin{aligned}
& \left(\int_{0}^{t} \int_{\Omega} u u_{t} d x d \tau\right)^{2} \leq \int_{0}^{t} \int_{\Omega}\left|u_{t}\right|^{2} d x d \tau \cdot \int_{0}^{t} \int_{\Omega}|u|^{2} d x d \tau \\
& \left(\int_{0}^{t} \int_{\Omega} \nabla u \nabla u_{t} d x d \tau\right)^{2} \leq \int_{0}^{t} \int_{\Omega}\left|\nabla u_{t}\right|^{2} d x d \tau \cdot \int_{0}^{t} \int_{\Omega}|\nabla u|^{2} d x d \tau,
\end{aligned}
$$

and

$$
\begin{aligned}
& 2 \int_{0}^{t} \int_{\Omega} u u_{t} d x d \tau \cdot \int_{0}^{t} \int_{\Omega} \nabla u \nabla u_{t} d x d \tau \\
& \leq 2\left(\int_{0}^{t} \int_{\Omega}\left|u_{t}\right|^{2} d x d \tau\right)^{\frac{1}{2}} \cdot\left(\int_{0}^{t} \int_{\Omega}|u|^{2} d x d \tau\right)^{\frac{1}{2}} \\
& \cdot\left(\int_{0}^{t} \int_{\Omega}\left|\nabla u_{t}\right|^{2} d x d \tau\right)^{\frac{1}{2}} \cdot\left(\int_{0}^{t} \int_{\Omega}|\nabla u|^{2} d x d \tau\right)^{\frac{1}{2}} \\
& \leq \int_{0}^{t} \int_{\Omega}\left|\nabla u_{t}\right|^{2} d x d \tau \cdot \int_{0}^{t} \int_{\Omega}|u|^{2} d x d \tau \\
&+\int_{0}^{t} \int_{\Omega}\left|u_{t}\right|^{2} d x d \tau \cdot \int_{0}^{t} \int_{\Omega}|\nabla u|^{2} d x d \tau
\end{aligned}
$$

Inserting (3.10)-(3.12) into (3.9), we find

$$
\begin{aligned}
G^{\prime \prime}(t) G(t) \geq & 2 \alpha\left(\int_{0}^{t} \int_{\Omega} u u_{t} d x d \tau\right)^{2}+2 \alpha\left(\int_{0}^{t} \int_{\Omega} \nabla u \nabla u_{t} d x d \tau\right)^{2} \\
& +4 \alpha \int_{0}^{t} \int_{\Omega} u u_{t} d x d \tau \cdot \int_{0}^{t} \int_{\Omega} \nabla u \nabla u_{t} d x d \tau \\
= & 2 \alpha\left[\int_{0}^{t} \int_{\Omega}\left(u u_{t}+\nabla u \nabla u_{t}\right) d x d \tau\right]^{2} \\
= & \frac{\alpha}{2}\left(\int_{0}^{t} G^{\prime \prime}(\tau) d \tau\right)=\frac{\alpha}{2}\left(G^{\prime}(t)-G^{\prime}(0)\right)^{2} .
\end{aligned}
$$

Thus, we obtain

$$
G^{\prime \prime}(t) G(t)-\frac{\alpha}{2}\left(G^{\prime}(t)-G^{\prime}(0)\right)^{2} \geq 0
$$

On the other hand, from (3.6), we know

$$
\lim _{t \rightarrow \infty} H(t)=+\infty
$$

This implies

$$
G^{\prime}(t)=\int_{\Omega}\left[|u|^{2}+|\nabla u|^{2}\right] d x \rightarrow+\infty, \quad t \rightarrow \infty .
$$

Hence, for $2<\beta<\alpha$ there exists a $T_{\beta}$, such that for all $t \geq T_{\beta}$

$$
\alpha\left(G^{\prime}(t)-G^{\prime}(0)\right)^{2} \geq \beta G^{\prime}(t)^{2} .
$$


By (3.14) and (3.16), we have

$$
G^{\prime \prime}(t) G(t)-\frac{\beta}{2} G^{\prime}(t)^{2} \geq 0, \quad t \geq T_{\beta}
$$

We consider the function $G(t)^{-q}$ for $0<q<\frac{\beta}{2}$, we see that

$$
\begin{aligned}
\left(G(t)^{-q}\right)^{\prime \prime} & =q G(t)^{-q-2}\left[(q+1) G^{\prime}(t)^{2}-G^{\prime \prime}(t) G(t)\right] \\
& \leq q G(t)^{-q-2}\left[\frac{2(q+1)}{\beta}-1\right] G^{\prime \prime}(t) G(t)<0, \quad t \geq T_{\beta} .
\end{aligned}
$$

Since a concave function must always lie below any tangent line, we see that $G(t)^{-q}$ reaches 0 in finite time as $t \rightarrow T^{-}$, where $T>T_{\beta}$. This means

$$
\lim _{t \rightarrow T^{-}} G(t)=+\infty
$$

or

$$
\lim _{t \rightarrow T^{-}} \int_{0}^{t} \int_{\Omega}\left(|u|^{2}+|\nabla u|^{2}\right) d x d \tau=+\infty
$$

Then the desired assertion immediately follows.

\section{Competing interests}

The authors declare that they have no competing interests.

\section{Authors' contributions}

All authors contributed to each part of this work equally and read and approved the final manuscript.

\section{Acknowledgements}

This work is supported by the NSF of China (11401122, 40890153), the Scientific Program (2008B080701042) of Guangdong Province.

Received: 12 March 2015 Accepted: 3 June 2015 Published online: 24 June 2015

\section{References}

1. Al'shin, AB, Korpusov, MO, Siveshnikov, AG: Blow-up in Nonlinear Sobolev Type Equations. De Gruyter Series in Nonlinear Analysis and Applications, vol. 15. de Gruyter, Berlin (2011)

2. Barenblatt, Gl, Bertsch, M, DalPasso, R, Ughi, M: A degenerate pseudoparabolic regularization of a nonlinear forward-backward heat equation arising in the theory of heat and mass exchange in stably stratified turbulent shear flow. SIAM J. Math. Anal. 24(6), 1414-1439 (1993)

3. Barenblatt, Gl, Azorero, JG, Pablo, AD, Vazquez, JL: Mathematical model of the non-equilibrium water-oil displacement in porous strata. Appl. Anal. 65(1-2), 19-45 (1997)

4. Furter, J, Grinfeld, M: Local versus nonlocal interactions in population dynamics. J. Math. Biol. 27(1), 65-80 (1989)

5. Cantrell, RS, Consner, C: Diffusive logistic equations with indefinite weights: population models in disrupted environments. SIAM J. Math. Anal. 22(4), 1043-1064 (1991)

6. Padron, $\mathrm{V}$ : Effect of aggregation on population recovery modeled by a forward-backward pseudoparabolic equation. Trans. Am. Math. Soc. 356(7), 2739-2756 (2004)

7. Rosenau, P: Evolution and breaking of the ion-acoustic waves. Phys. Fluids 31(6), 1317-1319 (1988)

8. Kabanin, VA: Solution of mixed problem for fourth order equation. Differ. Uravn. 8(1), 54-61 (1972)

9. Bakiyevich, NI, Shadrin, GA: Cauchy problem for an equation in filtration theory. Sb. Trudov Mosgospedinstituta. 7, 47-63 (1978)

10. Bai, F, Elliott, CM, Gardiner, A, Spence, A, Stuart, AM: The viscous Cahn-Hilliard equation. I: computations. Nonlinearity 8(2), 131-160 (1995)

11. Elliott, CM, Stuart, AM: Viscous Cahn-Hilliard equation. Il: analysis. J. Differ. Equ. 128(2), 387-414 (1996)

12. Zhao, HJ, Xuan, BJ: Existence and convergence of solutions for the generalized BBM-Burgers equations. Nonlinear Anal. TMA 28(11), 1835-1849 (1997)

13. Khudaverdiyev, Kl, Farhadova, GM: On global existence for generalized solution of one-dimensional non-selfadjoint mixed problem for a class of fourth order semilinear pseudo-parabolic equations. Proc. Inst. Math. Mech. Natl. Acad. Sci. Azerb. 31, 119-134 (2009) 
14. Grasselli, M, Petzeltova, H, Schimperna, G: Asymptotic behavior of a nonisothermal the viscous Cahn-Hilliard equation with inertial term. J. Differ. Equ. 239(1), 38-60 (2007)

15. Qu, CY, Cao, Y: Global existence of solutions for a viscous Cahn-Hilliard equation with gradient dependent potentials and sources. Proc. Indian Acad. Sci. Math. Sci. 123(4), 499-513 (2013)

16. Levine, HA: Some nonexistence and instability theorems for solutions of formally parabolic equation of the form $P u_{t}=-A u+\mathcal{F}(u)$. Arch. Ration. Mech. Anal. 51, 371-386 (1973)

17. Chadam, JM, Peirce, A, Yin, HM: The blow up property of solutions to some diffusion equations with localized nonlinear reactions. J. Math. Anal. Appl. 169(2), 313-328 (1992)

18. Budd, C, Dold, J, Stuart, A: Blow-up in a partial differential equation with conserved first integral. SIAM J. Appl. Math. 53(3), 718-742 (1993)

19. Wang, MX, Wang, YM: Properties of positive solutions for nonlocal reaction-diffusion problems. Math. Methods Appl. Sci. (Online) 19(4), 1141-1156 (1996)

20. Souplet, P: Blow-up in nonlocal reaction-diffusion equations. SIAM J. Math. Anal. 29(6), 1301-1334 (1998)

21. Souplet, P: Uniform blow-up profile and boundary behavior for diffusion equations with nonlocal nonlinear source. J. Differ. Equ. 153(2), 374-406 (1999)

22. Deng, WB, Liu, QL, Xie, CH: Blow up properties for a class of nonlinear degenerate diffusion equation with nonlocal source. Appl. Math. Mech. 24(11), 1362-1368 (2003)

23. Chen, YP, Liu, QL, Xie, CH: Blow-up for degenerate parabolic equations with nonlocal source. Proc. Am. Math. Soc. $132(1), 135-145(2003)$

24. Liu, QL, Chen, YP, Xie, CH: Blow-up for a degenerate parabolic equations with a nonlocal source. J. Math. Anal. Appl. 285(2), 487-505 (2003)

25. Song, JC: Lower bounds for blow-up time in a nonlocal reaction diffusion problem. Appl. Math. Lett. 24(5), 793-796 (2011)

26. Liu, DM, Mu, CL, Qiao, X: Lower bounds estimate for the blow-up time of a nonlinear nonlocal porous medium equation. Acta Math. Sci. 32(3), 1206-1212 (2012)

27. Liu, Y: Lower bounds for the blow-up time in a nonlocal reaction diffusion problem under nonlinear boundary conditions. Math. Comput. Model. 57(3-4), 926-931 (2013)

28. Fang, ZB, Yang, R, Chai, Y: Lower bounds estimate for the blow-up time for a slow diffusion equation with nonlocal source and inner absorption. Math. Probl. Eng. 2014, Article ID 764248 (2014)

29. Fang, ZB, Zhang, JY: Global and blow up solutions for the nonlinear $p$-Laplacian evolution equation with weighted nonlinear nonlocal boundary condition. J. Integral Equ. Appl. 26(2), 171-196 (2014)

30. Korpusov, MO, Sveshnikov, AG: Blow-up of solutions of a Sobolev-type equation with a nonlocal source. Sib. Mat. Zh. 46(3), 567-578 (2005)

31. Bouziani, A: Solvability of nonlinear pseudoparabolic equation with a nonlocal boundary condition. Nonlinear Anal. 55, 883-904 (2003)

32. Lions, JL: Quelques méthodes de résolutions des probléms aux limites non linéaires. Dunod, Paris (1969)

33. Escobedo, M, Herrero, M: A semilinear parabolic system in bounded domain. Ann. Mat. Pura Appl. 165, 315-336 (1993)

\section{Submit your manuscript to a SpringerOpen ${ }^{\ominus}$ journal and benefit from:}

- Convenient online submission

- Rigorous peer review

- Immediate publication on acceptance

- Open access: articles freely available online

- High visibility within the field

- Retaining the copyright to your article 